\title{
Actividad inotrópica inducida por el derivado carbamacepina-alquino en un modelo de corazón aislado y perfundido a flujo constante
}

\author{
Lauro Figueroa-Valverde ${ }^{1}$, Francisco Díaz-Cedillo², María López-Ramos, Elodia García-Cervera, \\ Karen Quijano-Ascencio' \\ 1 Laboratorio de Investigación en Ciencias Biológicas y Fármaco-Química, Facultad de Ciencias Químico- \\ Biológicas, Universidad Autónoma de Campeche, Campeche, México \\ 2 Laboratorio de Química Orgánica, Escuela Nacional de Ciencias Biológicas, Instituto Politécnico Nacional, \\ México, D.F., México
}

Introducción. Existen pocos datos con respecto a los efectos de la carbamacepina y sus derivados en el aparato cardiovascular; además, el mecanismo molecular y su sitio de acción celular no son claros.

Objetivo. Evaluar los efectos inducidos por el derivado carbamacepina-alquino sobre la presión de perfusión, la resistencia vascular y la presión ventricular izquierda.

Materiales y métodos. Los efectos de la carbamacepina y del derivado carbamacepina-alquino sobre la presión de perfusión, la resistencia vascular y la presión ventricular izquierda fueron evaluados en un modelo de corazón aislado de rata (Langendorff).

Resultados. Se encontró que: 1) el derivado carbamacepina-alquino $\left(1 \times 10^{-9} \mathrm{mM}\right)$ incrementa la presión de perfusión y la resistencia vascular en comparación con la carbamacepina $\left(1 \times 10^{-9} \mathrm{mM}\right)$; 2) los efectos del derivado carbamacepina-alquino $\left(1 \times 10^{-9}-1 \times 10^{-4} \mathrm{M}\right)$ sobre la presión intraventricular no fueron inhibidos por metoprolol o prazosina $\left(1 \times 10^{-6} \mathrm{mM}\right)$; 3$)$; el nifedipino $\left(1 \times 10^{-6} \mathrm{mM}\right)$ bloquea los efectos ejercidos por el derivado carbamacepina-alquino $\left(1 \times 10^{-9}-1 \times 10^{-4} \mathrm{M}\right)$ sobre la presión intraventricular, y 4) el efecto del derivado de carbamacepina $\left(1 \times 10^{-9} \mathrm{mM}\right)$ incrementa la concentración de calcio intracelular a través del tiempo (3 a 18 minutos); sin embargo, en presencia de nifedipino $\left(1 \times 10^{-6} \mathrm{mM}\right)$, este efecto disminuye significativamente $(p=0,005)$.

Conclusiones. La actividad ejercida por el derivado carbamacepina-alquino sobre la presión de perfusión, la resistencia vascular y la presión intraventricular, involucra la activación del canal de calcio de tipo L, lo que trae como consecuencia indirecta cambios en los niveles de calcio intracelular y, subsecuentemente, induce un efecto inotrópico positivo.

Palabras clave: carbamacepina, nifedipino, presión ventricular, resistencia vascular, corazón.

Inotropic activity induced by carbamazepine-alkyne derivative in an isolated heart model and perfused to constant flow

Introduction. Few data exist with respect to the effects of carbamazepine and its derivatives at cardiovascular level; furthermore, the molecular mechanisms and cellular site of action are still unclear.

Objective. The effects induced by carbamazepine-alquine derivative on perfusion pressure, vascular resistance and left ventricular pressure were evaluated.

Materials and methods. The effects of carbamazepine and carbamazepine-alquine on the perfusion pressure, vascular resistance and left ventricular pressure were examined in isolated rat hearts (Langendorff model).

Results. Four results were obtained: (1) The carbamazepine-alquine derivative $\left[10^{-9} \mathrm{mM}\right]$ increased the perfusion pressure and vascular resistance in comparison with the carbamazepine [10-9 $\mathrm{mM}$; (2) the effect of carbamazepine-alquine derivative $\left[10^{-9}-10^{-4} \mathrm{mM}\right]$ on left ventricular pressure not was inhibited by metoprolol or prazosin at a dose of $10^{-6} \mathrm{mM}$; (3) nifedipine [10-6 $\mathrm{mM}$ ] blocked the effects exerted by the carbamazepine-alquine derivative $\left[10^{-9}-10^{-4} \mathrm{mM}\right]$ on left ventricular pressure, and (4) the carbamazepine-alquine derivative at dose of $10^{-9} \mathrm{mM}$ increased the concentration of intracellular calcium over a time period of 3-18 min; nevertheless, in presence of nifedipine $\left[10^{-6} \mathrm{mM}\right]$ this effect was inhibited significantly ( $p=0.005)$.

Conclusions. The activity exerted by carbamazepine-alquine derivative on perfusion pressure, vascular resistance and left ventricular pressure involved activation of calcium channel type-L, brought indirectly changes in the intracellular calcium levels and subsequently induced a positive inotropic effect.

Key words: carbamazepine, nifedipine, ventricular pressure, heart, vascular resistance 
La insuficiencia cardiaca congestiva es uno de los principales problemas de salud a nivel mundial (1-3). Desde hace varias décadas se han usado diversos fármacos con características inotrópicas positivas para su tratamiento $(4,5)$, ejemplo de esto es el tratamiento con digoxina (inhibidor de la ATPasa); sin embargo, su estrecho margen terapéutico hace que su uso sea cada vez menor al disponer de otras alternativas terapéuticas (6-8).

En este sentido, hay datos que indican que la dobutamina (agonista adrenérgico) puede ser útil en la insuficiencia cardiaca grave asociada a cardiopatía isquémica, infarto de miocardio y choque cardiogénico; sin embargo, su pobre biodisponiblidad oral trae como consecuencia que la dobutamina sea administrada por vía intravenosa; además, se ha observado que su administración continua durante 24 a 72 horas conduce a la pérdida progresiva de su efectividad (9). Otros estudios (10), realizados en pacientes con insuficiencia cardiaca congestiva, en los que se compara el efecto de la dobutamina con respecto al efecto inducido por levosimendán (inotrópico positivo, sensibilizador de calcio), mostraron una mejoría en la función hemodinámica con respecto al grupo tratado con dobutamina. Cabe señalar que la administración de dosis altas de levosimendán en pacientes con episodios de infarto del miocardio, puede inducir cambios en el efecto inotrópico por inhibir la actividad de algunas fosfodiesterasas (11).

Por otro lado, es importante señalar que existe otro tipo fármacos que son inhibidores de la isoenzima III de las fosfodiesterasas (12-14); ejemplo de esto es la milrinona que, a dosis de $0,75 \mu \mathrm{g} / \mathrm{kg}$ por minuto en pacientes con insuficiencia cardiaca congestiva, tiene efectos benéficos, induciendo un aumento en el gasto cardiaco y reduciendo la resistencia vascular sistémica (15); sin embargo, puede aumentar la incidencia de cardiopatía isquémica y de arritmias ventriculares en una forma dependiente de la dosis (16), por lo que el manejo terapéutico de estos fármacos condiciona su uso a gran escala, lo que trae como consecuencia la búsqueda de otras alternativas terapéuticas

\footnotetext{
Correspondencia:

Lauro Figueroa, Laboratorio de Investigación en Ciencias Biológicas y Fármaco-Química, Facultad de Ciencias QuímicoBiológicas, Universidad Autónoma de Campeche, Campeche, México

Teléfono: (981) 811 9800, extensión 73006; fax: (981) 8119800, extensión 73002

lauro_1999@yahoo.com

Recibido: 28/07/10; aceptado:10/03/11
}

para el tratamiento de la insuficiencia cardiaca congestiva.

Una de las alternativas farmacológicas son los inhibidores de la enzima convertidora de angiotensina y espirolactona en pacientes con insuficiencia cardiaca congestiva (17), los cuales reducen los efectos de cardiopatía isquémica y la recurrencia de infartos (18-20). Sin embargo, existen datos que señalan que el abrupto incremento en la prescripción de espironolactona se asocia a un mayor número de hospitalizaciones de pacientes con hipercaliemia y una mayor morbimortalidad por esta causa (21).

Todos estos datos indican que existen varios fármacos con actividad inotrópica para el uso de insuficiencia cardiaca congestiva. Sin embargo, los diversos efectos que se generan por su administración en pacientes puede deberse a:

1) las diferentes dosis administradas,

2) las diversas vías de administración, o

3) los diferentes mecanismos moleculares involucrados en el efecto inducido por cada uno de los fármacos utilizados para el control.

Por lo tanto, en la búsqueda de nuevas alternativas terapéuticas para el tratamiento de la insuficiencia cardiaca congestiva, se han evaluado algunos compuestos con actividad inotrópica que en su estructura poseen un anillo azepínico; ejemplo de esto es el efecto inotrópico positivo inducido por compuesto JTV-519 in vitro (22). Aunado a esto, otros estudios indican que el derivado azepínico (MC1-154) induce efectos inotrópicos en un modelo de aorta de rata (23).

Por lo anteriormente descrito, los estudios enfocados a analizar los efectos de compuestos azepínicos con efectos inotrópicos positivos son relativamente escasos, además de que el mecanismo de acción por el cual ejercen su efecto no es muy claro. Por lo tanto, el objetivo de este estudio fue evaluar el efecto de un nuevo compuesto que contiene en su estructura química un anillo de azepina (carbamacepina-alquino), sobre la capacidad de los vasos coronarios y sobre la resistencia vascular, traducidos por cambios en la presión de perfusión y la presión ventricular en un modelo de corazón aislado de rata (modelo de Langendorff modificado) (figura 1) (24).

Además, se usaron como herramientas farmacológicas varias sustancias, entre las que se encuentran nifedipino (antagonista de canales de 


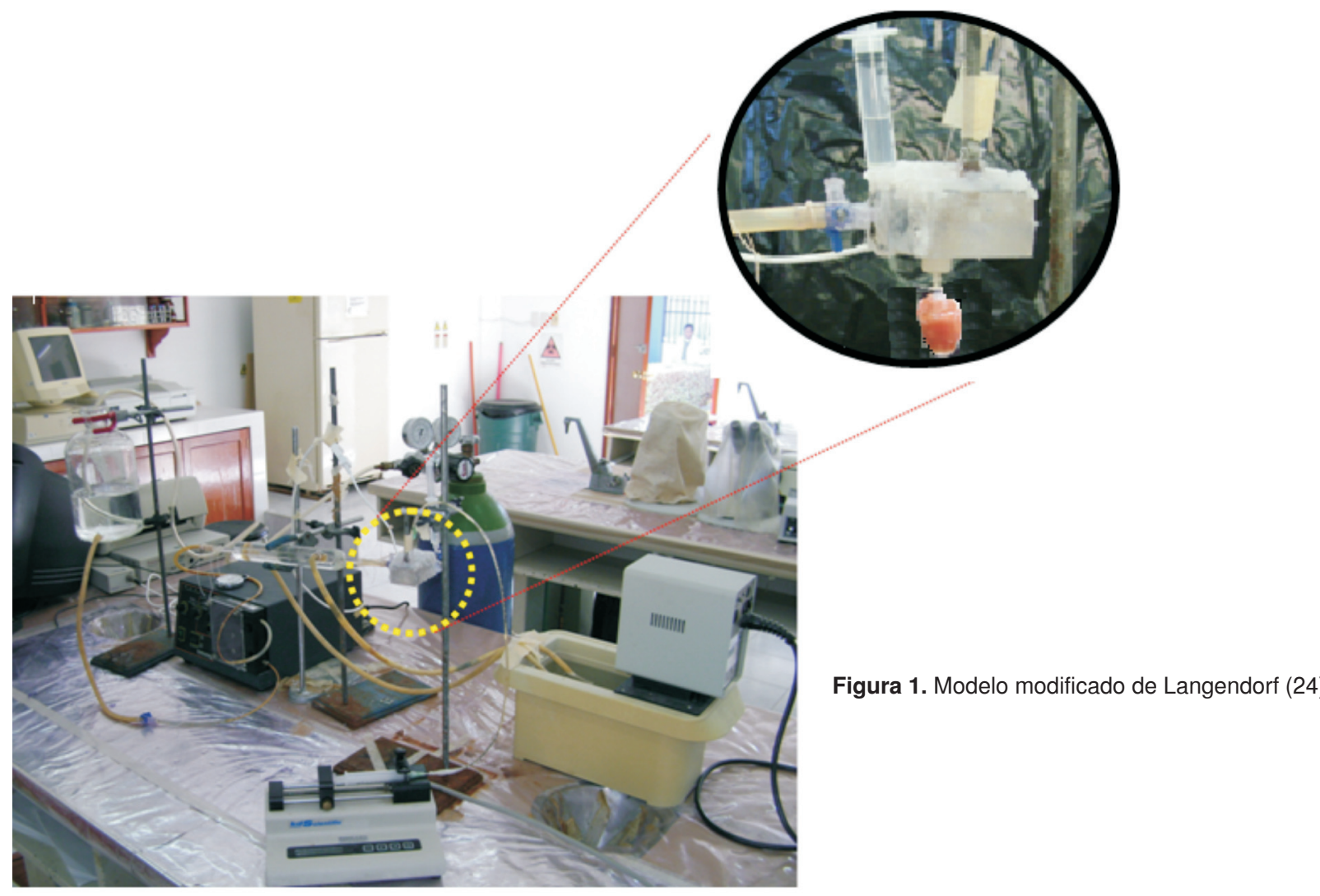

calcio de tipo-L) (25), metoprolol ( $\beta_{1}$-bloqueador) (26), prazosina ( $a_{1}$-bloqueador) (27) y cafeína (sensibilizador de canales de calcio de tipo-L) (28), para tratar de caracterizar el posible mecanismo involucrado en el efecto inotrópico ejercido por el derivado carbamacepina-alquino, tal como lo hacen otros tipos de sustancias que involucran indirectamente la síntesis y liberación de algunas substancias vasoactivasque ejercen indirectamente efectos sobre el tono vascular (29-31).

\section{Materiales y métodos}

\section{Material químico}

El derivado carbamacepina-alquino (figura 2) fue sintetizado usando técnicas previamente descritas (32). La carbamacepina y los otros reactivos fueron obtenidos de Sigma-Aldrich Chemical Co.

\section{Material biológico}

Todos los protocolos fueron aprobados por el Comité de Ética de la Universidad Autónoma de Campeche. Su aprobación se basó en la guía para cuidados y uso de animales de laboratorio, publicada por la National Academy of Sciences de los Estados Unidos, en 1996 (33). La cepa que se utilizó fue ratas Wistar (con un peso de 220 a $250 \mathrm{~g}$ ); antes<smiles>NC(=O)N1c2ccccc2C=Cc2ccccc21</smiles><smiles>CCCC/C=C/N(C(=O)N1c2ccccc2C=Cc2ccccc21)c1ccccc1</smiles>

Figura 2. Estructura química de carbamacepina (1) y el derivado carbamacepina-alquino (2).

de su experimentación se mantuvieron a una temperatura de $22{ }^{\circ} \mathrm{C}$ y fueron alimentadas con una dieta balanceada a base de purina y agua para mantenerlas en condiciones deseables.

\section{Diseño experimental}

La población de estudio fue de 45 ratas íntegras (machos, no castrados), de las cuales, se formaron cinco grupos para evaluar el efecto inducido por los compuestos estudiados en un modelo de corazón aislado y perfundido a flujo constante (10 $\mathrm{ml}$ por minuto). Es importante mencionar que cada corazón fue su propio control, para cada serie de experimentos el grupo etuvo compuesto por 9 animales. 


\section{Método de Langerdoff}

Los animales fueron anestesiados con pentobarbital por vía intraperitoneal $(50 \mathrm{mg} / \mathrm{kg})$. Después de la inducción de la anestesia, se extrajo el corazón del animal por medio de una laparatomía toracoabdominal, y se depositó en un vaso de precipitados que contenía solución de Krebs-Henseleit, la cual estaba compuesta de: $\mathrm{NaCl}(127 \mathrm{mM}), \mathrm{KCl}(6 \mathrm{mM})$, $\mathrm{CaCl}_{2}(1,8 \mathrm{mM}), \mathrm{NaH}_{2} \mathrm{PO}_{4}(1,2 \mathrm{mM}), \mathrm{MgSO}_{4}(1,2$ $\mathrm{mM}), \mathrm{NaHCO}_{3}(25 \mathrm{mM})$, dextrosa $(5 \mathrm{mM})$ y ácido pirúvico (2 mM).

Esta solución se ajustó a un pH de 7,4 (con $\mathrm{NaHCO}_{3}$ ) y se dejó que formara burbujas con una mezcla de gases $\left(5 \% \mathrm{CO}_{2}\right.$ y $\left.95 \% \mathrm{O}_{2}\right)$ a una temperatura de 35 a $36{ }^{\circ} \mathrm{C}$, regulada por un termostato. En este método, el corazón fue perfundido retrógradamente con la solución de Krebs-Henseleit, a través de una cánula insertada en la aorta (figura 1). Es importante mencionar que el tiempo de recuperación del corazón fluctúa entre 10 y 15 minutos. Después de este lapso, se hizo la evaluación farmacológica en tiempos no mayores de una hora.

\section{Presión de perfusión}

Los cambios en la presión de perfusión inducidos por la aplicación de la carbamacepina y el derivado carbamacepina-alquino, en ausencia de las sustancias involucradas en este estudio y en presencia de ellas, fueron cuantificados mediante una derivación de la cánula, la cual estaba insertada en cada corazón (figura 1), conectada a un transductor de presión (Statham ${ }^{\circledR}$ ) que, a su vez, estaba acoplado a un sistema de captura de datos computarizado (Biopac®). Cabe mencionar que la presión de perfusión intraórtica descrita por otros investigadores (34) es de $30 / 40 \mathrm{~mm} \mathrm{Hg}$ en ratas.

\section{Inducción de insuficiencia cardíaca}

La insuficiencia cardiaca se produjo mediante el método reportado por Mondragon et al. (35). Este proceso involucra la administración de una dosis controlada de pentobarbital sódico $(100 \mathrm{mg} /$ $\mathrm{kg}$ en dosis única), la cual se administró a través de la cánula insertada en la aorta. Es importante mencionar que cada corazón tiene un peso aproximado de 2 a $3 \mathrm{~g}$.

\section{Actividad inotrópica}

La actividad inotrópica del derivado carbamacepinaalquino se determinó usando los valores obtenidos de la presión ventricular izquierda. Para obtener dichos valores, en este método se hizo una incisión a nivel de la orejuela de la aurícula izquierda, por donde se introdujo un balón de látex delgado hasta el ventrículo izquierdo y distensible unido a una cánula de polietileno $(0,01 \mathrm{~mm}$ de diámetro), con el fin de medir la presión intraventricular izquierda, su primera derivada en relación con el tiempo $(d \mathrm{P} / d t)$. Antes de cada experimento, se determinó la relación presión-volumen del balón, y sólo se utilizó el balón con un volumen mayor o igual a $0,3 \mathrm{ml}$ y la presión es cero. El volumen de la pared del balón y del tubo dentro de él, se midieron por desplazamiento de agua con el balón completamente vacío (este volumen era de alrededor de $0,2 \mathrm{ml}$ ). Es importante mencionar que el balón estaba ligado a una cánula la cual, a su vez, fue conectada a un transductor de presión (Statham $\AA$ ) que, a su vez, estaba acoplado a un sistema de captura de datos computarizado (Biopac®).

\section{Evaluación de los efectos farmacológicos}

Primera fase. Evaluación del efecto inducido por la carbamacepina y el derivado carbamacepinaalquino sobre la presión de perfusión coronaria: se registró la presión de perfusión coronaria en tiempos de 3 a 18 minutos en ausencia (control) y en presencia de la carbamacepina y el derivado carbamacepina-alquino a dosis de $1 \times 10^{-9} \mathrm{mM}$ (dosis mínima). Los compuestos se disolvieron en metanol $(1 \mathrm{mg} / \mathrm{ml})$ y las diferentes concentraciones de trabajo se obtuvieron diluyendo esta solución concentrada con solución de K-H $(0,01 \%$, v/v).

Determinación del efecto ejercido por la carbamacepina y el derivado carbamacepina-alquino sobre la resistencia vascular: se obtuvo el registro del efecto sobre la resistencia vascular coronaria en condiciones de control, en presencia de la carbamacepina y el derivado carbamacepinaalquino, a una concentración de $1 \times 10^{-9} \mathrm{mM}$ (dosis mínima). Es importante mencionar que la carbamacepina y el derivado carbamacepinaalquino fueron perfundidos en lapsos no mayores de 18 minutos. La resistencia vascular coronaria se calculó dividiendo la presión de perfusión inducida por el flujo coronario por el el valor de flujo que la indujo ( $\mathrm{mm} \mathrm{Hg} / \mathrm{ml}$ por minuto) (36).

Segunda fase. Evaluación del efecto inducido por el derivado carbamacepina-alquino sobre receptores $\alpha_{1}$-adrenérgicos sobre la presión ventricular izquierda: en estos experimentos se administraron diferentes dosis $\left(1 \times 10^{-9}\right.$ a $\left.1 \times 10^{-4} \mathrm{mM}\right)$ del derivado carbamacepina-alquino y se registró la presión ventricular izquierda. La curva dosis-respuesta registrada (control) fue repetida bajo la perfusión 
continua de prasozín $\left(1 \times 10^{-6} \mathrm{mM}\right)$ en el mismo experimento. Cabe mencionar que la duración de la preincubación con prasozín fue de 10 minutos.

Determinación del efecto ejercido por el derivado carbamacepina-alquino sobre receptores $\beta_{1}$ adrenérgicos sobre la presión ventricular izquierda: se administraron diferentes dosis $\left(1 \times 10^{-9}\right.$ a $1 \times 10^{-4}$ $\mathrm{mM}$ ) del derivado carbamacepina-alquino y se registró la presión ventricular izquierda. La curva dosis-respuesta registrada (control) fue repetida bajo la perfusión continua de metoprolol $\left(1 \times 10^{-6}\right.$ $\mathrm{mM}$ ) en el mismo experimento. La preincubación con metoprolol fue de 10 minutos.

Efecto inducido por el derivado carbamacepinaalquino sobre la presión ventricular izquierda mediante canales de calcio de tipo-L: en estos experimentos se administraron diferentes dosis del derivado carbamacepina-alquino $\left(1 \times 10^{-9}\right.$ a $1 \times 10^{-4}$ $\mathrm{mM})$ y se registró la presión ventricular izquierda. La curva dosis-respuesta (control) fue repetida bajo perfusión continua de nifedipino $\left(1 \times 10^{-6} \mathrm{mM}\right)$ en el mismo experimento. Cabe señalar que la duración de la preincubación con nifedipino fue de 10 minutos.

Evaluación del efecto inducido por el derivado carbamacepina-alquino sobre la concentración de calcio intracelular: se evaluó el efecto del derivado carbamacepina-alquino a una dosis constante de $1 \times 10^{-9} \mathrm{mM}$ (dosis mínima) en tiempos de 3 a 18 minutos, y se determinaron los niveles de calcio intracelular. La curva dosis-respuesta (control) fue repetida bajo perfusión continua de nifedipino $\left(1 \times 10^{-6} \mathrm{mM}\right)$ en el mismo experimento.

Es importante mencionar que el calcio intracelular se evaluó mediante el método modificado reportado por Boe y Khan (37). En este método, se usaron alícuotas de $5 \mathrm{ml}$ obtenidas de la perfusión (solución biodegradada), a las cuales se les añadieron $4 \mathrm{ml}$ de ácido trifluoroacético (10 \%). La solución se mezcló por cinco minutos. Después, se agregó $1 \mathrm{ml}$ de $\mathrm{NaOH}(25 \%)$ con agitación constante. A esta mezcla se le añadió $1 \mathrm{ml}$ de fosfato trisódico y se agitó por una hora. La solución resultante se centrifugó (4.000 rpm) y el sobrenadante se separó de la fase acuosa. A la fase acuosa se le agregaron 5 gotas de una solución tampón de fosfatos $\left(\mathrm{Na}_{2} \mathrm{HPO}_{4} ; \mathrm{pH}=10\right)$ y 3 gotas de eriocromo negro. La mezcla resultante se tituló con una solución de EDTA (ácido etilen-diamino-tetraacético).

\section{Análisis estadístico}

Los valores obtenidos son expresados como media \pm error estándar de la media y los datos obtenidos fueron sometidos a la prueba t de Student y análisis de varianza (ANOVA) con factor de corrección de Bonferroni (38). Las diferencias se consideraron significativas cuando $p$ tenía un valor igual o menor de 0,05 .

\section{Resultados}

Fase 1. En este estudio se evaluó la actividad biológica de la carbamacepina y su derivado sobre la presión de perfusión coronaria. Los resultados obtenidos (figura 3) señalan que, con el incremento en el tiempo ( 3 a 18 minutos), en condiciones de control $(41,08$ a $42,84 \mathrm{~mm} \mathrm{Hg})$ y en presencia de carbamacepina $\left(1 \times 10^{-9} \mathrm{mM}\right)$, la presión de perfusión coronaria no varía significativamente (40,00 a 42,78 mm Hg). Sin embargo, en presencia del derivado carbamacepina-alquino $\left(1 \times 10^{-9} \mathrm{mM}\right)$, se incrementa significativamente $(48,88$ a 56,49 $\mathrm{mm} \mathrm{Hg} ; p=0,005$ ).

Además, los datos registrados de la evaluación del efecto del flujo coronario sobre la resistencia vascular coronaria (figura 4) en ausencia, así como en presencia de carbamacepina y el carbamacepina-alquino a una concentración de $1 \times 10^{-9}$ $\mathrm{mM}$, muestran que el derivado de androsterona incrementa significativamente $(p=0,005)$ la resistencia coronaria, en comparación con la carbamacepina y las condiciones de control.

Fase 2. Otros resultados obtenidos en experimentos alternativos donde se evaluó el efecto del derivado carbamacepina-alquino en dosis de $1 \times 10^{-9}$ a $1 \times 10^{-4} \mathrm{mM}$ sobre la presión ventricular izquierda, en ausencia y en presencia de metroprolol $\left(1 \times 10^{-6}\right.$ $\mathrm{mM}$ ), mostraron que el derivado de carbamacepina incrementa la presión de perfusión (figura 5) en forma dependiente de la dosis; sin embargo, en presencia del $\beta_{1}$-antagonista, este efecto no es inhibido.

Otros datos obtenidos de experimentos alternativos en los cuales se evaluó el efecto inducido por derivado carbamacepina-alquino sobre la presión ventricular izquierda, en ausencia y en presencia de prazosina $\left(1 \times 10^{-6} \mathrm{mM}\right)$, señalan que el efecto ejercido por el derivado carbamacepina-alquino $\left(1 \times 10^{-9} \mathrm{mM}\right.$ a $\left.1 \times 10^{-4} \mathrm{mM}\right)$ sobre la presión ventricular izquierda, no es bloqueado por el $a_{1}$ antagonista (figura 6).

Por otro lado, los resultados derivados de la evaluación del derivado carbamacepina-alquino sobre la presión ventricular izquierda, en ausencia y en presencia de nifedipino $\left(1 \times 10^{-6} \mathrm{mM}\right)$, señalan que el efecto inducido por el derivado carbamacepina- 


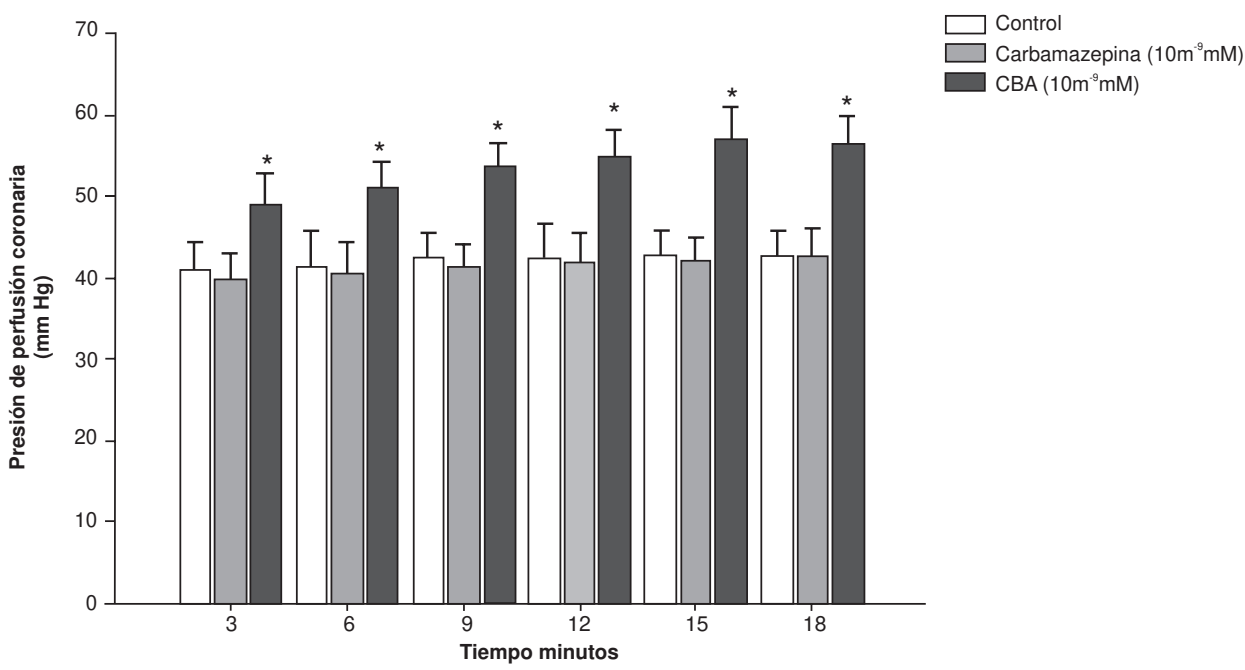

Figura 3. Efecto ejercido por la carbamacepina y el derivado carbamacepina-alquino sobre la presión de perfusión. Los resultados obtenidos señalan que con el incremento en el tiempo en condiciones de control y en presencia de carbamacepina, la presión de perfusión no varía significativamente; sin embargo, en presencia del derivado carbamacepina-alquino, se incrementa significativamente $(p=0,005)$. Cada barra representa la media \pm E.E.M. de nueve experimentos.

CBA: derivado carbamacepina-alquino

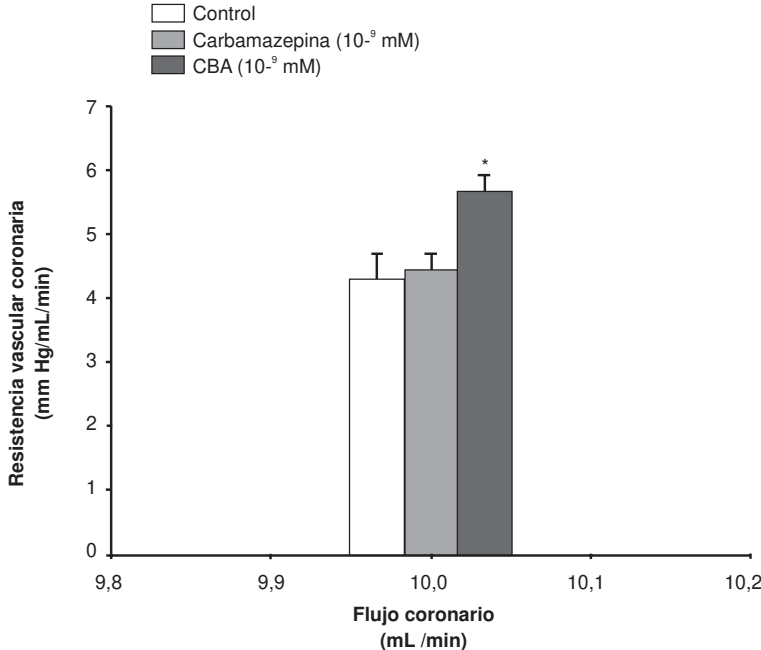

Figura 4. Actividad inducida por la carbamacepina y el derivado carbamacepina-alquino sobre la resistencia vascular coronaria. Los resultados indican que el derivado carbamacepinaalquino incrementa significativamente $(p=0,005)$ la resistencia vascular coronaria, en comparación con carbamacepina y las condiciones control. Cada barra representa la media \pm E.E.M. de nueve experimentos.

CBA: derivado carbamacepina-alquino

alquino $\left(1 \times 10^{-9}\right.$ a $\left.1 \times 10^{-4} \mathrm{mM}\right)$ sobre la presión de perfusión, es bloqueado significativamente $(p=0,005)$ por el antagonista del calcio (figura 7$)$.

En otros estudios en los que se evaluó el efecto inducido por la cafeína sobre la presión ventricular izquierda (figura 8), en presencia y en ausencia de nifedipino $\left(1 \times 10^{-9}\right.$ a $\left.1 \times 10^{-4} \mathrm{mM}\right)$, fue inhibido significativamente $(p=0,006)$. Finalmente, los resultados del

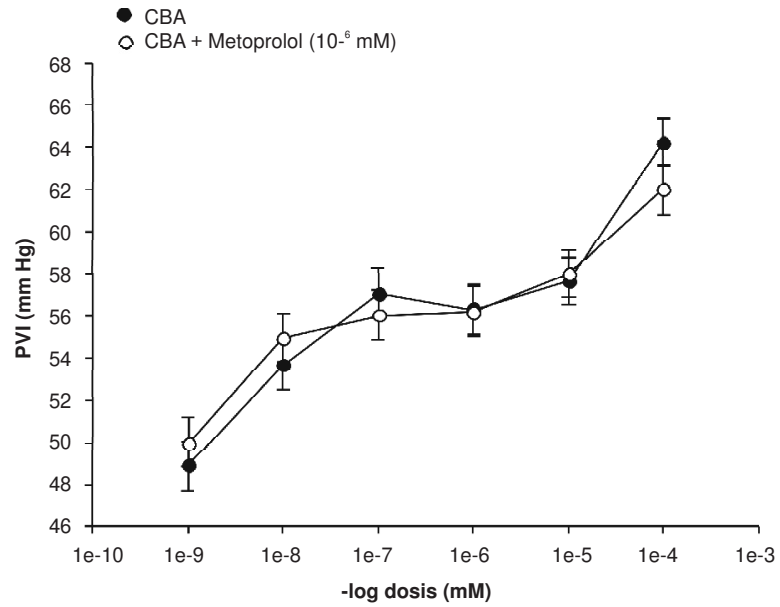

Figura 5. Evaluación del efecto inducido por el derivado carbamacepina-alquino sobre la presión ventricular izquierda, en ausencia y en presencia de metoprolol. Los registros obtenidos muestran que el derivado carbamacepina-alquino $(\bullet)$ incrementa la presión de perfusión en una manera que depende de la dosis. Además, en la gráfica se observa que, en presencia del $\beta_{1}$-bloqueador (o), este efecto no es inhibido. Cada punto representa la media \pm E.E.M. de nueve experimentos.

CBA: derivado carbamacepina-alquino

análisis de los niveles de calcio intracelular $\left(\mathrm{Ca}_{i}{ }^{++}\right)$ (cuadro 1), señalan que la administración del derivado carbamacepina-alquino a dosis de $1 \times 10^{-9}$ $\mathrm{mM}$ mostró incrementos en el calcio intracelular $\left(1,35 \times 10^{-3}\right.$ a $\left.2,03 \times 10^{-3} \mathrm{mM}\right)$ por aumentos en el tiempo (3 a 18 minutos); sin embargo, en presencia de nifedipino este efecto disminuyo significativamente $(p=0,005)$. 


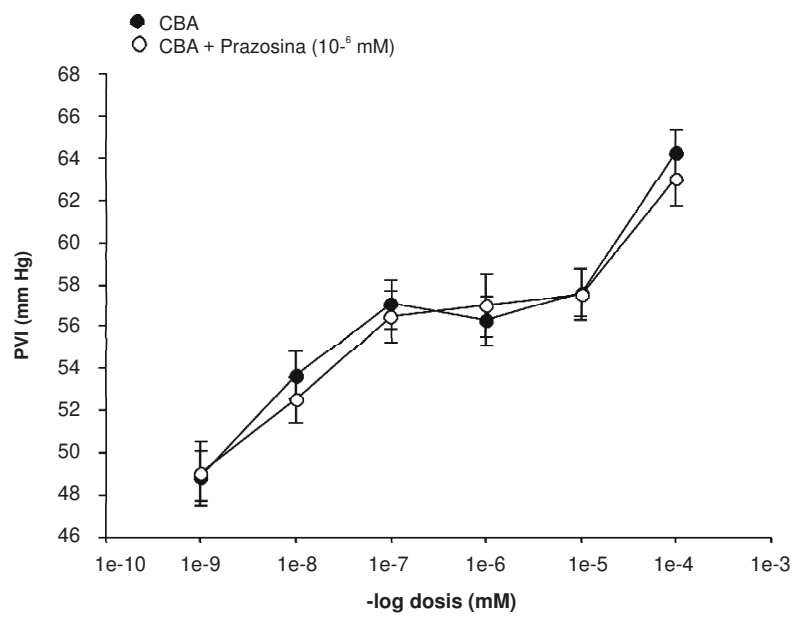

Figura 6. Actividad inducida por el derivado carbamacepinaalquino sobre la presión ventricular izquierda, en ausencia y en presencia de prazosina. Los datos obtenidos señalan que el aumento en la dosis de derivado carbamacepina-alquino (•) trae como consecuencia incrementos en la presión ventricular izquierda; sin embargo, en presencia del $a_{1}$-antagonista (o), este efecto no es bloqueado. Cada barra representa la media \pm E.E.M. de nueve experimentos.

CBA: derivado carbamacepina-alquino

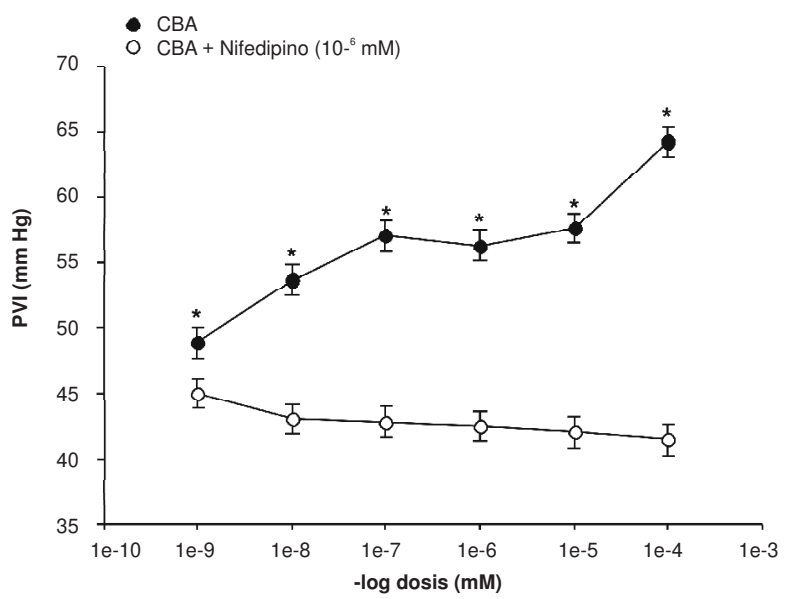

Figura 7. Efecto del derivado carbamacepina-alquino sobre la presión intraventricular izquierda, en ausencia y en presencia de nifedipino. Los resultados señalan que el efecto inducido por el análogo de carbamacepina $(\bullet)$ incrementa la presión de perfusión; sin embargo, este efecto es bloqueado significativamente $(p=0,005)$ por la nifedipino (o). Cada barra representa la media \pm E.E.M. de nueve experimentos.

CBA: derivado carbamacepina-alquino

\section{Discusión}

En este estudio se evaluó la actividad biológica de la carbamacepina y su derivado sobre la presión de perfusión coronaria, el cual se dividió en dos fases.

En la primera fase se evaluó el efecto inducido por la carbamacepina y el derivado de carbamacepina-

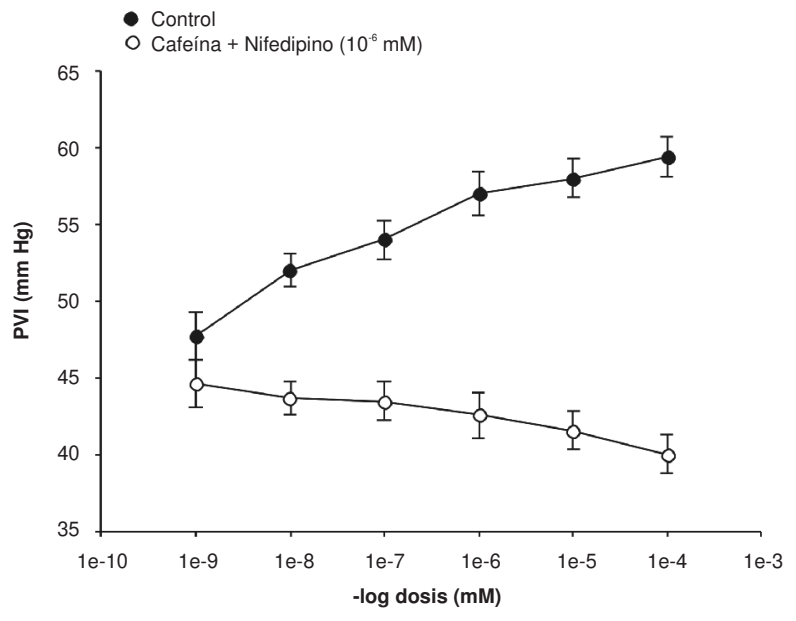

Figura 8. Evaluación del efecto inducido por la cafeína sobre la presión intraventricular izquierda. en ausencia y en presencia de nifedipino. Los registros obtenidos muestran que la cafeína $(\bullet)$ incrementa la presión de perfusión en una manera que depende de la dosis. Además, en la gráfica se observa que, en presencia de la nifedipino (o), este efecto es inhibido significativamente $(p=0,006)$. Cada punto representa la media \pm E.E.M. de nueve experimentos.

CBA: derivado carbamacepina-alquino

alquino sobre la capacidad de los vasos coronarios y sobre la resistencia coronaria, traducido por cambios en la presión de perfusión coronaria en corazón aislado de rata en un modelo de Langendorff (24). Vale la pena mencionar que nos pareció importante sólo mostrar la actividad ejercida por el derivado carbamacepina-alquino a una concentración de $1 \times 10^{-9} \mathrm{mM}$ (dosis mínima efectiva).

Los resultados obtenidos señalan que la presión de perfusión coronaria en condiciones control y en presencia de carbamacepina, no cambia con variaciones en el tiempo; sin embargo, en presencia del derivado carbamacepina-alquino, se incrementa significativamente. Estos datos indican que el derivado carbamacepina-alquino ejerce mayor efecto sobre la presión de perfusión coronaria en comparación con la carbamacepina, lo que podría traer como consecuencia modificaciones en el tono vascular coronario.

Pensando en esto, se evaluó el efecto inducido por la carbamacepina y su derivado sobre la resistencia vascular coronaria, traducido por cambios en la presión de perfusión. Los resultados obtenidos indican que la resistencia vascular se incrementa significativamente en presencia del derivado carbamacepina-alquino, en comparación con las condiciones de control y la carbamacepina. Es importante señalar que este fenómeno puede 
Cuadro 1. Determinación del calcio intracelular $\mathrm{Ca}_{\mathrm{i}}{ }^{++}$por la administración de CBA $\left(10^{-9} \mathrm{mM}\right)$ y $\mathrm{CBA}\left(10^{-9} \mathrm{mM}\right)$ más nifedipino $\left(10^{-6} \mathrm{mM}\right)$.

\begin{tabular}{lcc}
\hline & \multicolumn{2}{c}{$\mathrm{Ca}_{\mathbf{i}}{ }^{++}(\mathrm{mM})$} \\
\cline { 2 - 3 } $\begin{array}{l}\text { Tiempo } \\
\text { (minutos) }\end{array}$ & $\mathbf{C B A}$ & CBA más nifedipino* \\
\hline 3 & & $6,75 \times 10^{-4}$ \\
6 & $1,35 \times 10^{-3}$ & $6,75 \times 10^{-4}$ \\
9 & $1,35 \times 10^{-3}$ & $6,75 \times 10^{-4}$ \\
12 & $1,35 \times 10^{-3}$ & $6,75 \times 10^{-4}$ \\
15 & $2,03 \times 10^{-3}$ & $6,75 \times 10^{-4}$ \\
18 & $2,03 \times 10^{-3}$ & $6,75 \times 10^{-4}$ \\
\hline
\end{tabular}

${ }^{*} p=0,005$

CBA: derivado carbamacepina-alquino

estar condicionado por los diferentes mecanismos moleculares involucrados entre la carbamacepina (inhibidor de canales de sodio) (39) y su derivado.

Analizando la hipótesis anterior, así como datos reportados para otras sustancias que incrementan la presión de perfusión y la resistencia vascular coronaria (40) e inducen actividad inotrópica positiva (41), se ha encontrado que traen como consecuencia variaciones en el flujo sanguíneo (42) por medio del sistema adrenérgico. En este estudio, se realizaron experimentos en corazones con insuficiencia cardiaca, para tratar de caracterizar el efecto inducido por el derivado carbamacepina-alquino mediante receptores adrenérgicos. Los resultados mostraron que la actividad sobre la presión ventricular izquierda inducida por el derivado carbamacepina-alquino, no fue bloqueada por el metoprolol ( $\beta_{1}$-antagonista); por lo tanto, estos datos nos indicaron que el mecanismo molecular involucrado en la actividad ejercida por el derivado carbamacepina-alquino no era por vía $\beta$-adrenérgica.

Sin embargo, para corroborar que el sistema a-drenérgico no estaba involucrado en el mecanismo molecular por el cual el derivado carbamacepina-alquino ejerce su efecto, se realizaron experimentos alternativos usando como herramienta farmacológica a la prazosina, bajo las mismas condiciones que en el caso del metoprolol. Los resultados indican que, en presencia del $a_{1}$ antagonista, el efecto inducido por el derivado carbamacepina-alquino sobre la presión ventricular izquierda no fue inhibido, lo cual sugirió que no es la vía molecular por la cual esta sustancia ejerce su actividad farmacológica.

Por lo tanto, en la búsqueda del mecanismo molecular por el cual el derivado carbamacepina- alquino induce su efecto y analizando la actividad biológica ejercida por otros fármacos (43) los cuales actúan estimulando los depósitos intracelulares de calcio y la activación de la proteincinasa C lo que trae como consecuencia indirecta un incremento en el flujo de calcio intracelular a través de canales de calcio de tipo L Con base en esto, en este estudio se hicieron experimentos en los cuales se evaluó la actividad ejercida por el derivado carbamacepina-alquino sobre la presión ventricular izquierda, en ausencia y presencia nifedipino (antagonista de canales de calcio de tipo-L). Los resultados obtenidos indican que el efecto inducido por el derivado carbamacepinaalquino sobre la presión ventricular izquierda fue bloqueado significativamente por nifedipino. Estos resultados nos permiten postular que la actividad ejercida por el derivado involucra incrementos en la concentración de calcio intracelular mediante la activación de canales de calcio de tipo-L, como lo hacen otros tipos de sustancias (44).

Para evaluar esta premisa, en este estudio se usó como herramienta farmacológica a la cafeína. Es importante mencionar que existen reportes que indican que la cafeína incrementa indirectamente la concentración de calcio intracelular y la presión sanguínea (28). Por lo tanto, se realizaron experimentos alternativos en los que se evaluó el efecto de la cafeína en una forma dependiente de la dosis, en ausencia y en presencia de nifedipino.

Los resultados mostraron que el efecto ejercido por la cafeína incrementa la presión ventricular izquierda. Sin embargo, en presencia de nifedipino este efecto fue bloqueado significativamente. Estos resultados señalan que los efectos inducidos por la cafeína son similares a los efectos ejercidos por el derivado carbamacepina-alquino. Esos datos confirman que la actividad inducida por el derivado 
carbamacepina-alquino sobre la presión ventricular izquierda incrementa indirectamente los niveles de calcio intracelular mediante la activación de canales decalcio de tipo Lmediante un mecanismo molecular no genómico. Sin embargo, para complementar esta premisa, se realizaron experimentos en los que se evaluó la concentración de calcio intracelular por la administración de derivado carbamacepina-alquino en forma dependiente de la dosis, en ausencia y en presencia de nifedipino. Los resultados señalan que los niveles de calcio intracelular disminuyeron en presencia de nifedipino, lo que vuelve a corroborar que el efecto inducido por el derivado carbamacepina-alquino afecta indirectamente la concentración de este ión intracelular que está involucrado en cambios de la presión intraventricular, como lo señalan algunos investigadores $(45,46)$.

En conclusión, la actividad ejercida por el derivado carbamacepina-alquino sobre la presión de perfusión, la resistencia vascular coronaria y la presión intraventricular, involucra la activación del canal de calcio de tipo $\mathrm{L}$, lo que trae indirectamente cambios en los niveles de calcio intracelular y, subsecuentemente, un efecto inotrópico positivo. Todos estos datos indican que el derivado de carbamacepina podría ser considerado como un buen fármaco para el tratamiento de insuficiencia cardiaca congestiva; sin embargo, es válido señalar que se deben hacer evaluaciones toxicológicas en posteriores etapas experimentales para descartar posibles riesgos.

\section{Conflicto de intereses}

No se tiene ningún conflicto de intereses con autoridades públicas o privadas para su publicación.

\section{Financiación}

Este proyecto fue financiado por CONACyT con No. de registro 52542.

\section{Referencias}

1. Cleland J, Clark A. Has the survival of the heart failure population changed? Lessons from trials. Am J Cardiol. 1999;83:112D-9.

2. Lobos JM, Díaz S, Redondo R. Evaluación de la insuficiencia cardíaca desde la consulta de atención primaria. Form Med Contn Aten Prim. 1997;4:10-23.

3. Boix MR, Almazán IJ, Medrano AM. Mortalidad por insuficiencia cardíaca en España, 1977-1998. Rev Esp Cardiol. 2002;55:219-26.

4. Lechat P, Packer M, Chalon S, Cucherat M, Arab T, Boissel JP. Clinical effects of beta-adrenergic blockade in chronic heart failure: A meta-analysis of double-blind, placebocontrolled, randomized trials. Circulation. 1998;98:1184-91.

5. Carson PE. Blocker therapy in heart failure: Pathophysiology and clinical results. Curr Probl Cardiol. 1999;24:426-60.

6. Mendéz R. Doscientos años de digital. Arch Inst Cardiol Mex.1986;56:339-48.

7. Tischler $\mathbf{M}$, Smith $\mathbf{T}$. Digitalis: Its current place in the treatment of heart failure. Modern Conc Cardiovasc Dis. 1990;59:67-72.

8. Ahmed A, Rich M, Fleg J, Zile M, Young J, Kitzman D, et al. Effects of digoxin on morbidity and mortality in diastolic heart failure. Circulation. 2006;114:397-403.

9. Mayes J, Carter C, Adams JE. Inotropic therapy in the home care setting: Criteria, management, and implications. J Intraven Nurs. 1995;18:301-6.

10. Follath F, Cleland JG, Just H, Papp JG, Scholz H, Peuhkurinen K. Steering Committee and Investigators of the Levosimendan Infusion versus Dobutamine (LIDO) Study. Efficacy and safety of intravenous levosimendan compared with dobutamine in severe low-output heart failure (the LIDO study): A randomized double-blind trial. Lancet. 2002;360:196-202.

11. Papp JG. Introduction: Positive inotropy by calcium sensitization-an evolving approach for the treatment of endstage heart failure. Am J Cardiol. 1999;83:1-3.

12. Corvalán H, Casanegra P, Chamorro S, Jalil M, Valenzuela P. Comparación de los efectos clínicos y neurohumorales de milrinona y captopril en pacientes con insuficiencia cardíaca crónica. Bol Cardiol Chile. 1988;7:289-300.

13. Bregagnollo E, Fortes AH, Cicogna AC. Avaliação dos efeitos inotrópicos e vasodilatadores do lactato de milrinona em pacientes com cardiomiopatia dilatada e insuficiência cardíaca grave. Arq Bras Cardiol. 1999;72:149-54.

14. Cosín AJ, Hernández MA. Ensayos clínicos en insuficiencia cardíaca. Rev Esp Cardiol. 2001;54: 22-31.

15. Hoffman TM, Wernovsky G, Atz AM, Kulik TJ, Nelson DP, Chang AC. Efficacy and safety of milrinone in preventing low cardiac output syndrome in infants and children after corrective surgery for congenital heart disease. Circulation. 2003;107:996-1002.

16. Gol V, Barroso P. Farmacología de la insuficiencia cardíaca. Farm Hosp. 1996;20:149-56.

17. The CONSENSUS Trial Study Group. Effects of enalapril on mortality in severe congestive heart failure. Results of the Cooperative North Scandinavian Enalapril Survival Study (CONSENSUS). N Engl J Med. 1987;316:1429-35.

18. The SOLVD Investigators. Effect of enalapril on survival in patients with reduced left ventricular ejection fractions and congestive heart failure. N Engl J Med. 1991;325:293-302.

19. Tamargoa J, López-Sendón J. Avances en el tratamiento de la insuficiencia cardíaca. IV - Bases y evidencias clínicas de los efectos de los nuevos tratamientos farmacológicos en la insuficiencia cardíaca. Rev Esp Cardiol. 2004;57:44764.

20. Pitt B, Zannad F, Remme WJ, Cody R, Castaigne A, Pérez A. The effect of spironolactone on morbidity and mortality in patients with severe heart failure. Randomized 
aldactone evaluation study investigators. N Engl J Med. 1999;341:709-17.

21. Juurlink D, Mamdani MM, Lee DS, Kopp A, Austin PO, Laupacis A. Rates of hyperkalemia after publication of the randomized aldactone evaluation study. $\mathrm{N}$ Engl J Med.

22. Inagaki K, Kihara Y, Izumi T, Sasayama S. The cardioprotective effects of a new 1,4-benzothiazepine derivative, JTV519, on ischemia/reperfusion-induced Ca2+ overload in isolated rat hearts. Cardiovasc Drugs Ther. 2000;14:489-95.

23. Shibata S, Satake N, Hester RK, Kurahashi K, Ito M. The mode of vasoinhibitory action of a pyridazione derivative (MCl-154), a new cardiotonic agent, on contractile responses induced by alpha-adrenoceptor agonists and $45 \mathrm{Ca}$ influx in isolated vascular smooth muscles. Eur $\mathrm{J}$ Pharmacol. 1988;12;145:113-21

24. Figueroa-Valverde L, Díaz-Ku E, Díaz-Cedillo $F$, Baqueiro-Bricaire C, Camacho-Luis A. Efectos del danazol y hemisuccinato de danazol sobre la presión de perfusión y resistencia vascular. Acta Bioquím Clín Latinoam. 2010;44:37-45.

25 Henry PD. Comparative pharmacology of calcium antagonists: Nifedipine, verapamil and diltiazem. Am J Cardiol. 1980;46:1047-58.

26. Bengtsson C, Johnsson G, Regårdh C. Plasma levels and effects of metoprolol on blood pressure and heart rate in hypertensive patients after an acute dose and between two doses during long-term treatment. Clin Pharmacol Ther. 1975;17:400-8

27. Graham R, Oates H, Stoker L, Stokes G. Alpha blocking action of the antihypertensive agent, prazosin. J Pharmacol Exper Ther. 1977;201:747-52.

28. Sitsapesan R, Williams AJ. Mechanisms of caffeine activation of single calcium-release channels of sheep cardiac sarcoplasmic reticulum. J Physiol. 1990;423:425-39.

29. Figueroa-Valverde L, Ceballos-Reyes G, Díaz-Cedillo F, Camacho-Luis A, López-Ramos M, MaldonadoVelázquez G. Biological activity of progesteronedihydropyridimidine derivative on perfusion pressure and coronary resistance in isolated rat heart. African $\mathrm{J}$ Pharm Pharmacol. 2010;4:170-7.

30. Figueroa-Valverde L, Díaz-Cedillo F, Camacho-Luis A, Díaz-Ku E, Marvin R. Actividad inducida por androsterona y hemisuccinato de androsterona sobre la presión de perfusión y la resistencia vascular. Biomédica. 2009;29:62534.

31. Ceballos RG, Figueroa VL, Rubio I, Gallo G, García A, Martínez A, et al. Acute and nongenomic effects of testosterone on isolated and perfused rat heart. J Cardiovasc Pharmacol. 1999;33:691-7.

32. Figueroa-Valverde L, Díaz-Cedillo F, Camacho-Luis A, López-Ramos M, García-Cervera E. Design and synthesis of carbamazepine-alkyne conjugate as antidiabetic agent: Study of chemical descriptors ( $\log P$ and $\pi)$. Chem Asian J. 2010;22:7057-64.
33. Institute of Laboratory Animal, Resources Commission on Life Sciences, National Research Council. Guide for the care and use of laboratory animals. Seventh edition. Washington, D.C.: National Academies Press; 1996. p. $1-240$.

34. Nestler J, Beer N, Jakubowicz D, Colombo C, Beer Neely $\mathrm{J}$, Liebermeister et al. Effect of pressure development on oxygen consumption by isolated rat heart. Am J Physiol. 1967;212:804-14.

35. Mondragón VL, Narváez TJ, Zarco OZ, Tenorio LF, Pastelín HP. Estudio de la relación entre las características electromoleculares de los digitálicos y su acción farmacológica. Arch Cardiol Mex. 2003;73:11-7.

36. Figueroa-Valverde L, Luna H, Castillo-Henkel C, MuñozGarcía O, Morato-Cartagena T, Ceballos-Reyes G. Synthesis and evaluation of the cardiovascular effects of two, membrane impermeant, macromolecular complexes of dextran-testosterone. Steroids. 2002;67:611-9.

37. Boe JH, Khan BS. Colorimetrie determination of blood calcium. J Biol Chem. 1928;81:1-8.

38. Hocht C, Opezzo J, Gorzalczany S, Bramuglia G, Tiara C. Una aproximación cinética y dinámica de metildopa en ratas con coartación aórtica mediante microdiálisis. Rev Argent Cardiol. 1999;67:769-73.

39. Willow M, Gonoi T, Catterall WA. Voltage clamp analysis of the inhibitory actions of diphenylhydantoin and carbamazepine on voltage-sensitive sodium channels in neuroblastoma cells. Mol Pharm.1985;27:549-58.40. Figueroa-Valverde L, Díaz-CedilloF, Diaz-Ku E, CamachoLuis A. Effect induced by hemisuccinate of pregnenolone on perfusion pressure and vascular resistance in isolated rat heart. African J Pharm Pharmacol. 2009;3:234-41.

41. Broadley JK. An analysis of the coronary vascular responses to catecholamines, using a modified Langendorff heart preparation. Br J Pharmacol. 1970;40:617-29.

42. Parratt RJ. The evidence for a-adrenoceptors in the coronary circulation and their possible relevance to the physiological regulation of myocardial blood flow. Basic Res Cardiol. 1981;76:485-93.

43. Szokodi I, Kinnunen P, Tavi P, Weckstrom M, Toth M, Ruskoaho H. Evidence for cAMP-independent mechanisms mediating the effects of adrenomedullin, a new inotropic peptide. Circulation. 1998;97:1062-70.

44. Schramm M, Towart TG, Franckowiak G. Novel dihydropyridines with positive inotropic action through activation of $\mathrm{Ca}^{2+}$ channels. Nature. 1983;303:535-7.

45. Beuckelmann DJ, Nabauer M, Erdmann E. Intracellular calcium handling in isolated ventricular myocytes from patients with terminal heart failure. Circulation. 1992;85:1046-55

46. Lang R, Fellner S, Neumann A, Bushinsky D, Boroe K. Left ventricular contractility varies directly with blood ionized calcium. Ann Intern Med. 1988;108:524-9. 